\title{
Feasibility of quantitative perfusion CMR in patients with poor left ventricular function
}

\author{
Eva Sammut", Niloufar Zarinabad, Zhong Chen, Reza Razavi, Eike Nagel, Amedeo Chiribiri \\ From 16th Annual SCMR Scientific Sessions \\ San Francisco, CA, USA. 31 January - 3 February 2013
}

\section{Background}

First pass perfusion CMR is a well-validated technique for the detection of inducible myocardial ischaemia. However, limited data are available on the feasibility of first pass perfusion CMR and quantitative deconvolution analysis in the context of reduced LV function.

The aim of this study was to assess effects of reduced LV function and LV dilatation on the characteristics of signal in the left ventricle (arterial input function, AIF) and in the myocardium (MSI), and to test the feasibility of quantitative perfusion analysis with Fermi deconvolution in this cohort of patients.

\section{Methods}

We retrospectively identified fifteen consecutive patients who had undergone perfusion CMR in the presence of impaired LV function (left ventricular ejection fraction $(\mathrm{LVEF})<50 \%)$ at our centre. Five healthy volunteers were enrolled in the study as control subjects. Perfusion data were obtained using a pre-bolus technique and $0.075 \mathrm{mmol} / \mathrm{kg}$ Gadobutrol (Gadovist, Schering, Germany) injected at $4 \mathrm{ml} /$ minute followed by a $20 \mathrm{ml}$ saline flush. Images were acquired on a Philips Achieva 3T (TX) system, equipped with a 32-channel cardiac phased array receiver coil (Philips, Best, Netherlands). First-pass perfusion images were acquired using a saturation recovery gradient echo method (TR/TE $3.0 \mathrm{~ms} /$ $1.0 \mathrm{~ms}$, flip-angle $15^{\circ}$; effective k-t SENSE acceleration 3.8, spatial resolution $1.2 \times 1.2 \times 10 \mathrm{~mm}$, saturation-recovery delay $120 \mathrm{~ms}$ ).

The following parameters were evaluated from stress perfusion series in order to assess the quality of the data and assess the effect of abnormal cardiac structure and function: time to peak, peak value and maximum slope

of the AIF and of the MSI. Quantitative analysis was performed by Fermi deconvolution.

\section{Results}

Detailed results are reported in Table. The left ventricular (LV) systolic function was lower, with higher end-diastolic (LV EDV) and end-systolic volumes (LV ESV) in the patient compared with the normal group.

Both the AIF and the MSI were reduced in peak amplitude, maximum upslope and time to peak in patients compared with controls. However, the ratio between the peak of AIF and MSI and the time to peak of the AIF and MSI was constant between patients and controls, suggesting the feasibility of deconvolution quantitative analysis on both groups.

Fermi deconvolution showed a trend towards an overall lower perfusion rate in normal segments in the patients group $(2.2 \pm 1.2 \mathrm{ml} / \mathrm{g} / \mathrm{min})$ compared with controls $(2.7$ $\pm 0.8 ; \mathrm{p}=0.2$ ) however non-significant. Reduced perfusion rate was found in visually abnormal segments in patients $(1.6 \pm 0.8 ; \mathrm{p}=0.03)$.

\section{Conclusions}

Our results show that reduced cardiac function modulates the characteristics of both the AIF and MSI. However, the relationship between AIF and MSI remains unaffected. Fermi deconvolution analysis appears applicable to both groups, supporting the feasibility of first pass perfusion CMR as a clinical tool in patients with reduced LV function.

\section{Funding}

None. 


\section{Table 1 Showing values across patient and control group}

\begin{tabular}{cccc}
\hline & Patient group & Control group & p value \\
\hline LVEF (\%) & 40.1 (SD 9.4) & 67.0 (SD 3.7) & $<.0001$ \\
LV EDV (ml) & 108.3 (SD 34.0) & 75.6 (SD 7.8) & 0.0030 \\
LV ESV (ml) & 67.1 (SD 31.1) & 25.2 (SD 4.1) & 0.0001 \\
Peak AIF & 389.4 (SD 328.2) & 1239.7 (SD 637.8) & 0.0379 \\
Peak MSI & 113.5 (SD 83.4) & 358.4 (SD 199.4) & 0.0497 \\
Peak AlF / peak MSI & 3.6 (SD 1.4) & 3.7 (SD 0.7) & 0.9054 \\
Time to peak LVAIF (seconds) & 9.1 (SD 3.2) & 6.1 (SD 0.8) & 0.0052 \\
Time to peak MSI (seconds) & 12.1 (SD 4.5) & 7.6 (SD 1.0) & 0.0025 \\
Time to peak MSI / time to peak AlF (seconds) & 3.1 (SD 3.7) & 1.5 (SD 0.5) & 0.1250 \\
\hline
\end{tabular}

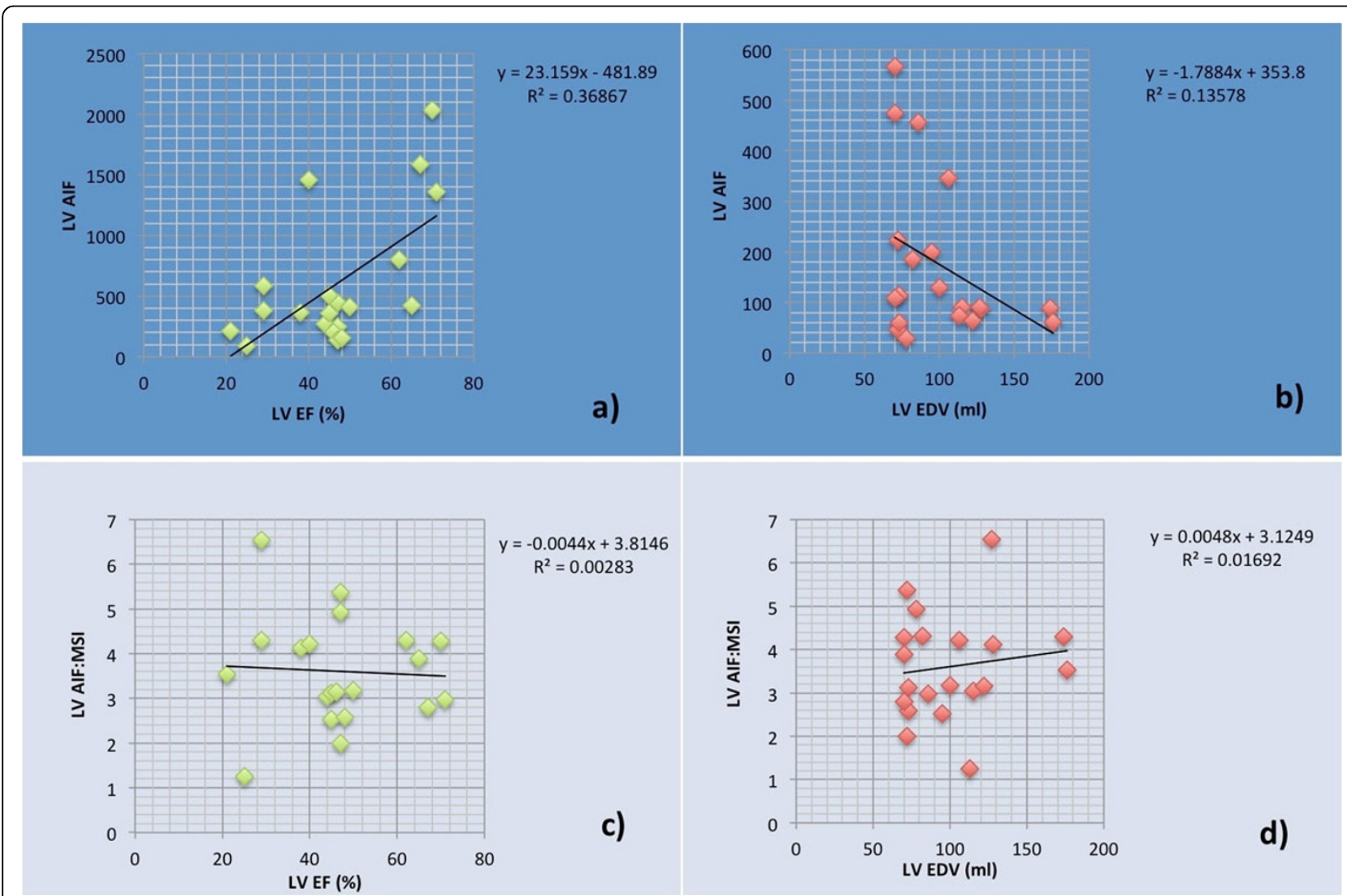

Figure 1 a) LVEF vs peak LV AIF, b) LV EDV vs LV AIF, c) LV EF vs peak LVAIF/peak MSI, d) LV EDV vs peak LVAIF/peak MSI.

Published: 30 January 2013

doi:10.1186/1532-429X-15-S1-E60

Cite this article as: Sammut et al:: Feasibility of quantitative perfusion

CMR in patients with poor left ventricular function. Journal of

Cardiovascular Magnetic Resonance 2013 15(Suppl 1):E60.

\section{Submit your next manuscript to BioMed Central} and take full advantage of:

- Convenient online submission

- Thorough peer review

- No space constraints or color figure charges

- Immediate publication on acceptance

- Inclusion in PubMed, CAS, Scopus and Google Scholar

- Research which is freely available for redistribution 